\title{
Examination of the PCICE Method in the Nearly Incompressible, as well as Strictly Incompressible, Limits *
}

\author{
Ray A. BERRY** and Richard C. MARTINEAU*** \\ ** Idaho National Laboratory \\ Multiphysics Methods Group, P.O. Box 1625, Idaho Falls, Idaho 83415-3840 USA \\ E-mail:Ray.Berry@inl.gov \\ *** Idaho National Laboratory \\ Multiphysics Methods Group, P.O. Box 1625, Idaho Falls, Idaho 83415-3840 USA
}

\begin{abstract}
The conservative-form, pressure-based PCICE numerical method (Martineau and Berry, 2004) (Berry, 2006), recently developed for computing transient fluid flows of all speeds from very low to very high (with strong shocks), is simplified and generalized. Though the method automatically treats a continuous transition of compressibility, three distinct, limiting compressibility regimes are formally defined for purposes of discussion and comparison with traditional methods - the strictly incompressible limit, the nearly incompressible limit, and the fully compressible limit. The PCICE method's behavior is examined in each limiting regime. In the strictly incompressible limit the PCICE algorithm reduces to the traditional MAC-type method with velocity divergence driving the pressure Poisson equation. In the nearly incompressible limit the PCICE algorithm is found to reduce to a generalization of traditional incompressible methods, i.e. to one in which not only the velocity divergence effect, but also the density gradient effect is included as a driving function in the pressure Poisson equation. This nearly incompressible regime has received little attention, and it appears that in the past, strictly incompressible methods may have been conveniently applied to flows in this regime at the expense of ignoring a potentially important coupling mechanism. This could be significant in many important flows; for example, in natural convection flows resulting from high heat flux. In the fully compressible limit or regime, the algorithm is found to reduce to an expression equivalent to density-based methods for high-speed flow.
\end{abstract}

Key words: Compressible, Incompressible, Strictly Incompressible, Nearly Incompressible, Fully Compressible

\section{Introduction}

Single phase flows of practical import for current and future nuclear reactor systems cover a wide range of flow speeds and compressibilities. Seldom do they belong exclusively to the restrictive class of strictly incompressible flow. Most reactor flows are of a mixed nature meaning that they possess regions dominated by, for example, nearly incompressible flow as well as regions dominated by strongly compressible effects. Or, for transients, during part of the time compressible effects will dominate while at different time the flow will be nearly incompressible. To be able to handle such flows, the pressure-corrected variant of the implicit continuous-fluid Eulerian (ICE) (Harlow and Amsden, 1971), or 
PCICE numerical method has been developed and presented (Martineau and Berry, 2004) as a finite element method, PCICE-FEM, for computing fluid flows of all speeds from low subsonic or nearly incompressible to high supersonic compressible. PCICE is a predictor-corrector method for approximating the solution of the conservative form of the Euler/Navier-Stokes equations:

$$
\begin{aligned}
& \frac{\partial \rho}{\partial t}+\nabla \cdot \rho \vec{u}=0 \\
& \frac{\partial \rho \vec{u}}{\partial t}+\nabla \cdot(\rho \vec{u} \otimes \vec{u})=-\nabla p+\nabla \cdot \tau \\
& \frac{\partial \rho e_{t}}{\partial t}+\nabla \cdot \rho \vec{u} h_{t}=\nabla \cdot(\tau \cdot \vec{u})+\nabla \cdot k \nabla T+i(T)
\end{aligned}
$$

where $\rho, \vec{u}, p$, and $T$ represent the fluid mass density, velocity, pressure, and temperature, respectively. In these equations, $\tau$ is the shear stress, $e_{t}=e+\frac{\vec{u} \cdot \vec{u}}{2}$ is the total energy density (where $e$ is the internal energy density), $h_{t}=\frac{\rho e_{t}+p}{\rho}$ is the specific total enthalpy, and $i(T)$ is a temperature-dependent energy source term. Fourier's law for thermal conduction has been assumed with $k$ denoting the thermal conductivity. These equations represent the balance of mass, momentum, and total energy, respectively. Because the PCICE method is not restricted to any specific equation of state, the general functional form

$$
p=f(\rho, e)
$$

will be utilized throughout this development.

The objectives of this short note are two-fold. First, as in (Berry, 2006), it presents a simplified, yet generalized, description of the PCICE method, independent of specific spatial discretizations and equations of state. Second, the behavior of the PCICE method is examined in the strictly incompressible limit, the nearly incompressible limit $(c \Delta t>>$, where $c$ is the acoustic wave speed, $\Delta t$ represents the time resolution of interest, and $l$ represents the characteristic length of interest), as well as in the fully compressible limit $(c \Delta t<<l)$.

\section{PCICE Algorithm}

The pressure-corrected implicit continuous-fluid Eulerian, or PCICE algorithm (Martineau and Berry, 2004)(Berry, 2006), is an ideal basis with which to construct a fully coupled unified physics computer analysis code. This scheme, developed for all-speed compressible and nearly incompressible flows, improves upon previous pressure-based methods in terms of accuracy and numerical efficiency and gives a wider range of applicability. Because of the need to simulate flows with shocks it is essential that both the governing equations and their discretized approximations be in conservative form (Lax and Wendroff, 1960)(Leveque, 1990). Unlike other ICE variants that have been proposed in the past, most of which are entirely or partially in primitive form, the PCICE algorithm solves the conservative form of the governing equations.

Other researchers have coupled, to varying extent, energy effects into ICE-type algorithms (Casulli and Greenspan, 1984)(Liles and Reed, 1978)(Westbrook, 1978) (Xiao, et al., 1997), but most have utilized, entirely or partially, nonconservative forms which led to algorithms which are restricted to smooth transient solutions (no discontinuity waves) or to steady solutions with sonic- and lower speeds. Patnaik, et. al. 
(Patnaik, et al., 1987) developed a "barely implicit" ICE-type algorithm in conservative form which primarily couples the momentum and energy equations, similar to that of Cassuli and Greenspan (Casulli and Greenspan, 1984). The PCICE algorithm efficiently incorporates an even higher degree of implicitness into a very general conservative framework which can be utilized with either finite difference, finite volume, or finite element spatial representations. In the PCICE algorithm, the total energy equation is sufficiently coupled to the pressure Poisson equation to avoid iteration between the pressure Poisson equation and the pressure-correction equations. The pressure Poisson equation then has the time-advanced internal energy information it requires to yield an accurate implicit pressure. At the end of a time step, the conserved values of mass, momentum, and total energy are all pressure-corrected. As a result, the iterative process usually associated with pressure-based schemes is not required. This aspect has been found advantageous when computing transient compressible flows, including flows with significant energy deposition, chemical reactions, or phase change.

The pressure-based PCICE solution algorithm is composed of two fractional steps. The first fractional step is composed of an explicit predictor-corrector Heun's method applied without the pressure gradient term present in the momentum equation (projection-type approach). In the second fractional step an implicit pressure correction is employed - an elliptic Poisson equation is solved for new-time pressures followed by an explicit correction with the new pressures. The pressure, momentum, and density in the governing hydrodynamic equations are treated in an implicit fashion. The so-called mass-momentum coupling is obtained by substituting the momentum balance equations into the mass conservation equation to eliminate time-advanced momentum-density (or mass flux) as an unknown. The time rate of density change in the mass conservation equation is then expressed in terms of pressure and internal energy change by employing the equation of state. These substitutions result in a single second-order elliptic differential equation in terms of pressure (pressure Poisson equation). This semi-implicit treatment has two advantages over explicit schemes. First, the acoustic component from the explicit time step size stability criteria is removed, thus eliminating the time integration stiffness that results from slow flows. Second, the pressure obtained with this semi-implicit treatment corrects the momentum to satisfy mass conservation requirements. This allows nearly incompressible flows to be simulated with compressible flow equations, which can be used to simulate flows from very low speeds to supersonic, including mixed flows with all flow speeds present.

Though our original description of the PCICE algorithm was in the context of a finite-element based method, PCICE-FEM (Martineau and Berry, 2004), with an ideal gas equation of state, it can be generally implemented within the context of other spatial discretization methods (finite-difference, finite-volume, grid-free, etc.) (Berry, 2006). Therefore, the description of the PCICE algorithm given here will be kept free of specific spatial discretizations and equations of state.

\section{Temporal Discretization}

The PCICE algorithm is a predictor-corrector method for solving the following time discretization of balance Eqns. (1), (2), and (3) for mass, momentum, and energy, respectively (for illustration, here simplified to the Euler equations):

$$
\rho^{n+1}=\rho^{n}-\frac{\Delta t}{2} \nabla \cdot\left[(\rho \vec{u})^{n+1}+(\rho \vec{u})^{n}\right]
$$




$$
\begin{aligned}
& (\rho \vec{u})^{n+1}=(\rho \vec{u})^{n}-\Delta t \vec{\nabla} \cdot(\rho \vec{u} \otimes \vec{u})^{n+\frac{1}{2}}-\frac{\Delta t}{2} \vec{\nabla}\left(p^{n+1}+p^{n}\right) \\
& \left(\rho e_{t}\right)^{n+1}=\left(\rho e_{t}\right)^{n}-\frac{\Delta t}{2} \vec{\nabla} \cdot\left[(\rho \vec{u})^{n+1} h_{t}^{n+1}+(\rho \vec{u})^{n} h_{t}^{n}\right] .
\end{aligned}
$$

The divergence term in the momentum equation is at a partially time-advanced level obtained by utilizing an explicit two-step, modified Heun's method which will be described subsequently. In the original PCICE-FEM method (Martineau and Berry, 2004), an efficient Taylor-Galerkin method was used, however the modified Heun's method exhibits lower phase errors near discontinuities. These equations are approximated with the following fractional two-step process.

\subsection{Fractional Step 1}

The first fractional step solves a portion of Eqns. (5)-(7), with a predictor step:

$$
\begin{aligned}
& \rho^{*}=\rho^{n}-\Delta t \nabla \cdot(\rho \vec{u})^{n} \\
& (\rho \vec{u})^{*}=(\rho \vec{u})^{n}-\Delta t \vec{\nabla} \cdot(\rho \vec{u} \otimes \vec{u})^{n} \\
& \left(\rho e_{t}\right)^{*}=\left(\rho e_{t}\right)^{n}-\Delta t \vec{\nabla} \cdot(\rho \vec{u})^{n} h_{t}^{n} \\
& \vec{u}^{*}=\frac{(\rho \vec{u})^{*}}{\rho^{*}} \quad e_{t}^{*}=\frac{\left(\rho e_{t}\right)^{*}}{\rho^{*}} \quad e^{*}=e_{t}^{*}-\frac{\vec{u}^{*} \cdot \vec{u}^{*}}{2} \\
& p^{*}=f\left(\rho^{*}, e^{*}\right) \quad h_{t}^{*}=\frac{\left(\rho e_{t}\right)^{*}+p^{*}}{\rho^{*}}
\end{aligned}
$$

followed by a corrector step:

$$
\begin{aligned}
\rho^{* *}= & \rho^{n}-\frac{\Delta t}{2} \nabla \cdot\left[(\rho \vec{u})^{*}+(\rho \vec{u})^{n}\right] \\
= & \rho^{*}-\frac{\Delta t}{2} \nabla \cdot\left[(\rho \vec{u})^{*}-(\rho \vec{u})^{n}\right] \\
(\rho \vec{u})^{* *} & =(\rho \vec{u})^{n}-\frac{\Delta t}{2} \vec{\nabla} \cdot\left[(\rho \vec{u} \otimes \vec{u})^{*}+(\rho \vec{u} \otimes \vec{u})^{n}\right] \\
& =(\rho \vec{u})^{*}-\frac{\Delta t}{2} \vec{\nabla} \cdot\left[(\rho \vec{u} \otimes \vec{u})^{*}-(\rho \vec{u} \otimes \vec{u})^{n}\right] \\
\left(\rho e_{t}\right)^{* *} & =\left(\rho e_{t}\right)^{n}-\frac{\Delta t}{2} \vec{\nabla} \cdot\left[(\rho \vec{u})^{*} h_{t}^{*}+(\rho \vec{u})^{n} h_{t}^{n}\right] \\
& =\left(\rho e_{t}\right)^{*}-\frac{\Delta t}{2} \vec{\nabla} \cdot\left[(\rho \vec{u})^{*} h_{t}^{*}-(\rho \vec{u})^{n} h_{t}^{n}\right] \\
\vec{u}^{* *}= & \frac{(\rho \vec{u})^{* *}}{\rho^{* *}} \quad e_{t}^{* *}=\frac{\left(\rho e_{t}\right)^{* *}}{\rho}=e_{t}^{* *}-\frac{\vec{u}^{* *} \cdot \vec{u}^{* *}}{2} \\
p^{* *}= & f\left(\rho^{* *}, e^{* *}\right) \quad h_{t}^{*}=\frac{\left(\rho e_{t}\right)^{* *}+p^{* *}}{\rho}
\end{aligned}
$$


It is important that the quantities $\rho,(\rho \vec{u})$, and $\left(\rho e_{t}\right)$, for both the predictor $(*)$ and corrector $(* *)$ steps, be advanced with high-order monotonic algorithms such as FCT, TVD, ENO, etc. or that they be smoothed with another appropriate smoother such as the variable diffusion method of Swanson and Turkel (Swanson and Turkel, 1992) used in the finite element version PCICE-FEM (Martineau and Berry, 2004).

As with projection-type schemes, the pressure gradient is not included in the partial momentum balance Eqns. (9) and (13). Instead, it will be included implicitly in the next fractional step. Therefore the time step stability restriction for this fractional step is the satisfaction of the material Courant condition, or the Courant condition based on flow speed. If the shear stress, heat transfer, or energy source terms are included and treated explicitly, a stable time step based on these terms may be too restrictive. To obtain additional stability with larger time steps, such terms can be treated implicitly, either here in this fractional step, or in an additional (subsequent or previous) fractional step.

\subsection{Fractional Step 2}

This fractional step seeks to solve the following portion of the original discretized Eqns. (5)-(7) to obtain new time pressure.

\section{Momentum}

$$
\begin{aligned}
(\rho \vec{u})^{n+1} & =(\rho \vec{u})^{* *}-\frac{\Delta t}{2} \nabla\left(p^{n+1}+p^{n}\right) \\
& =(\rho \vec{u})^{* *}-\frac{\Delta t}{2} \nabla\left(p^{n+1}-p^{n}\right)-\Delta t \nabla p^{n}
\end{aligned}
$$

Mass

$$
\rho^{n+1}=\rho^{* *}-\frac{\Delta t}{2} \nabla \cdot\left[(\rho \vec{u})^{n+1}-(\rho \vec{u})^{*}\right]
$$

\section{Total Energy}

$$
\left(\rho e_{t}\right)^{n+1}=\left(\rho e_{t}\right)^{* *}-\frac{\Delta t}{2} \nabla \cdot\left[(\rho \vec{u})^{n+1} h_{t}^{n+1}-(\rho \vec{u})^{*} h_{t}^{*}\right]
$$

Eqn. (16) is required to achieve the target discretization, and is obtained by subtracting Eqn. (13) from Eqn. (6) using

$$
(\rho \vec{u} \otimes \vec{u})^{n+\frac{1}{2}}=\frac{1}{2}\left[(\rho \vec{u} \otimes \vec{u})^{*}+(\rho \vec{u} \otimes \vec{u})^{n}\right] .
$$

Similarly, Eqn. (17) is obtained by subtracting Eqn. (12) from (5) and Eqn. (18) results from subtracting Eqn. (14) from (7).

The solution to Eqns. (16) - (18) is accomplished in a couple of steps, first by constructing a pressure Poisson equation which is solved to obtain pressures at the new-time level, then correcting the dependent variables with these new-time pressures. 
The pressure Poisson equation is constructed by first substituting Eqn. (16) into Eqn. (5)

$$
\rho^{n+1}=\rho^{n}-\frac{\Delta t}{2} \nabla \cdot\left[(\rho \vec{u})^{* *}-\frac{\Delta t}{2} \nabla \cdot\left(p^{n+1}-p^{n}\right)-\Delta t \nabla p^{n}+(\rho \vec{u})^{n}\right]
$$

or with rearrangement

$$
\begin{aligned}
\rho^{n+1}-\rho^{n}=\frac{\Delta t^{2}}{4} \nabla & \cdot \nabla\left(p^{n+1}-p^{n}\right)+\frac{\Delta t^{2}}{2} \nabla \cdot \nabla p^{n} \\
- & \frac{\Delta t}{2} \nabla \cdot\left[(\rho \vec{u})^{* *}+(\rho \vec{u})^{n}\right]
\end{aligned} .
$$

From the equation of state (EOS)

$$
p=f(\rho, e)
$$

one can obtain

$$
\delta \rho=\frac{1}{\frac{\partial f}{\partial \rho}} \delta p-\frac{\frac{\partial f}{\partial e}}{\frac{\partial f}{\partial \rho}} \delta e
$$

where $\delta$ just indicates a perturbation or change in a quantity. This leads to the simple approximation

$$
\rho^{n+1}-\rho^{n} \approx \frac{1}{\left(\frac{\partial f}{\partial \rho}\right)^{* *}}\left(p^{n+1}-p^{n}\right)-\frac{\left(\frac{\partial f}{\partial e}\right)^{* *}}{\left(\frac{\partial f}{\partial \rho}\right)^{* *}}\left(e^{* *}-e^{n}\right)
$$

The pressure Poisson equation, in terms of $\delta p=p^{n+1}-p^{n}$, is finally obtained by substituting Eqn. (20) into Eqn. (19) giving

$$
\begin{aligned}
\frac{1}{\left(\frac{\partial f}{\partial \rho}\right)^{* *}\left(p^{n+1}-p^{n}\right)-\frac{\Delta t^{2}}{4} \nabla \cdot \nabla\left(p^{n+1}-p^{n}\right)} & =\frac{\left(\frac{\partial f}{\partial e}\right)^{* *}}{\left(\frac{\partial f}{\partial \rho}\right)^{* *}}\left(e^{* *}-e^{n}\right) \\
& -\frac{\Delta t}{2} \nabla \cdot\left[(\rho \vec{u})^{* *}+(\rho \vec{u})^{n}\right] \\
& +\frac{\Delta t^{2}}{2} \nabla \cdot \nabla p^{n} .
\end{aligned}
$$

Numerical solution of this equation by an efficient, elliptic partial differential equation solver yields the new pressure distribution, $p^{n+1}$.

The other dependent variables are then updated, or corrected, with the new-time pressures as follows, in order: 


\section{Momentum}

$(\rho \vec{u})^{n+1}=(\rho \vec{u})^{* *}-\frac{\Delta t}{2} \nabla\left[p^{n+1}+p^{n}\right]$

Mass

$\rho^{n+1}=\rho^{* *}-\frac{\Delta t}{2} \nabla \cdot\left[(\rho \vec{u})^{n+1}-(\rho \vec{u})^{*}\right]$

Total Energy

$h_{t}^{n+1}=\frac{\left(\rho e_{t}\right)^{* *}+p^{n+1}}{\rho^{n+1}}$

$\left(\rho e_{t}\right)^{n+1}=\left(\rho e_{t}\right)^{* *}-\frac{\Delta t}{2} \nabla \cdot\left[(\rho \vec{u})^{n+1} h_{t}^{n+1}-(\rho \vec{u})^{*} h_{t}^{*}\right]$

\section{Compressible and Incompressible Limits}

The objective here is to identify and examine various compressible limiting forms of the PCICE algorithm. Dividing the pressure Poisson Eqn. (21) by $\Delta t$, noting that the isentropic sound speed $c$ is given by

$c^{2}=\frac{p}{\rho^{2}}\left(\frac{\partial f}{\partial e}\right)+\left(\frac{\partial f}{\partial \rho}\right)$,

and introducing

$\frac{1}{\left(\frac{\partial f}{\partial \rho}\right)}=\frac{A}{c^{2}}$ where $A=\left[1+\frac{p\left(\frac{\partial f}{\partial e}\right)}{\rho^{2}\left(\frac{\partial f}{\partial \rho}\right)}\right]$,

transforms pressure Poisson equation into

$$
\begin{aligned}
\frac{A^{* *}}{\left(c^{* *}\right)^{2}} \frac{p^{n+1}-p^{n}}{\Delta t} & =\frac{\Delta t}{4} \nabla^{2} p^{n+1}+\frac{A^{* *}\left(\frac{\partial f}{\partial e}\right)^{* *}}{\left(c^{* *}\right)^{2}} \frac{e^{* *}-e^{n}}{\Delta t} \\
& -\frac{1}{2} \nabla \cdot\left[(\rho \vec{u})^{* *}+(\rho \vec{u})^{n}\right] \\
& +\frac{\Delta t}{4} \nabla^{2} p^{n} .
\end{aligned}
$$

If Eqn. (26) is multiplied by a characteristic length, $l$, and a characteristic time,

$$
\begin{aligned}
\tau_{c} \frac{p^{n+1}-p^{n}}{\Delta t}= & \frac{l c^{* *} \Delta t}{4 A^{* *}} \nabla^{2} p^{n+1}+\tau_{c}\left(\frac{\partial f}{\partial e}\right)^{* *} \frac{e^{* *}-e^{n}}{\Delta t} \\
& -\frac{l c^{* *}}{2 A^{* *}} \nabla \cdot\left[(\rho \vec{u})^{* *}+(\rho \vec{u})^{n}\right] \\
& +\frac{l c^{* *} \Delta t}{4 A^{* *}} \nabla^{2} p^{n} .
\end{aligned}
$$

The characteristic time $\tau_{c}$ approximates the time it takes an acoustic wave (traveling with velocity $c^{* *}$ to propagate the distance $(l)$ characterizing the portion of our solution domain of interest and effecting the solution change. While incompressible fluids don't 
physically exist, such a mathematical model can be conceived by supposing the time resolution of interest corresponds to $\Delta t$. Thus if the case is desired in which $\tau_{c}<<\Delta t$, then necessarily the resolution of the physical acoustic waves which produce the solution change is not of interest. Furthermore, a characteristic time $\tau_{u}=\frac{l}{|\vec{u}|}$ can be identified which approximates the time it takes to advect the solution a distance $l$.

\subsection{Nearly Incompressible Limit}

In the nearly incompressible limit $\tau_{c}<<\Delta t<\tau_{u}$, or in other words $c^{*} \Delta t>>l$, which implies that $\frac{\tau_{c}}{\Delta t} \rightarrow 0$ and the pressure Poisson Eqn. (27) effectively reduces to

$$
\nabla^{2} p^{n+1}=\frac{2}{\Delta t} \nabla \cdot\left[(\rho \vec{u})^{* *}+(\rho \vec{u})^{n}\right]-\nabla^{2} p^{n}
$$

Obviously the nearly incompressible limit also implies that $\frac{|\vec{u}|}{c^{*}}<<\frac{|\vec{u}| \Delta t}{l}<1$, or the Mach Number $<<1$.

\subsection{Strictly Incompressible Limit}

Substituting the gradient of the equation of state

$$
\nabla \rho=\frac{1}{\left(\frac{\partial f}{\partial \rho}\right)} \nabla p-\frac{\left(\frac{\partial f}{\partial e}\right)}{\left(\frac{\partial f}{\partial \rho}\right)} \nabla e=\frac{A}{c^{2}} \nabla p-\frac{A\left(\frac{\partial f}{\partial e}\right)}{c^{2}} \nabla e
$$

into the expanded third term on the right side of Eqn. (27) gives

$$
\begin{aligned}
-\frac{l c^{* *}}{2 A^{* *}} \nabla \cdot\left[(\rho \vec{u})^{* *}+(\rho \vec{u})^{n}\right]= & -\frac{l c^{* *}}{2 A^{* *}}\left[\vec{u}^{* *} \cdot \nabla \rho^{* *}+\rho^{* *}\left(\nabla \cdot \vec{u}^{* *}\right) \vec{u}^{n} \cdot \nabla \rho^{n}+\rho^{n}\left(\nabla \cdot \vec{u}^{n}\right)\right] \\
= & -\frac{\tau_{c}}{2} \vec{u}^{* *} \cdot\left[\nabla p^{* *}-\left(\frac{\partial f}{\partial e}\right)^{* *} \nabla e^{* *}\right]-\frac{l c^{* *}}{2 A^{* *}} \rho^{* *}\left(\nabla \cdot \vec{u}^{* *}\right) \\
& -\frac{\tau_{c}^{n}}{2}\left(\frac{A^{n}}{A^{* *}}\right)\left(\frac{c^{* *}}{c^{n}}\right) \vec{u}^{n} \cdot\left[\nabla p^{n}-\left(\frac{\partial f}{\partial e}\right)^{n} \nabla e^{n}\right] \\
& -\frac{l c^{* *}}{2 A^{* *}} \rho^{n}\left(\nabla \cdot \vec{u}^{n}\right)
\end{aligned}
$$

In the strictly incompressible limit, $\tau_{c} \rightarrow 0, \tau_{c}^{n} \rightarrow 0$ (because sound speeds are infinite), so Eqn. (30) becomes

$$
\begin{aligned}
-\lim _{\tau_{c} \rightarrow 0} \lim _{\tau_{c}^{n} \rightarrow 0} \frac{l c^{* *}}{A^{* *}} \nabla \cdot\left[(\rho \vec{u})^{* *}+(\rho \vec{u})^{n}\right]= \\
-\frac{l c^{* *}}{2 A^{* *}}\left[\rho^{* *}\left(\nabla \cdot \vec{u}^{* *}\right)+\rho^{n}\left(\nabla \cdot \vec{u}^{n}\right)\right]
\end{aligned}
$$

and the pressure Poisson Eqn. (27) reduces to

$$
\nabla^{2} p^{n+1}=\frac{2}{\Delta t}\left[\rho^{* *}\left(\nabla \cdot \vec{u}^{* *}\right)+\rho^{n}(\nabla \cdot \vec{u})\right]-\nabla^{2} p^{n}
$$


Note that $\tau_{c} \rightarrow 0$ and $\tau_{c}{ }^{n} \rightarrow 0$ imply, physically, that the characteristic length scale of interest $l$ is very small relative to the sound speed, as opposed to considering incompressible flow to possess an infinite sound speed, as some researchers choose. If $\nabla \cdot \vec{u}^{n}=0$, Eqn. (32) can be compared directly with the corresponding, traditional MAC-type pressure Poisson equation (Harlow and Welch, 1965), without convective terms,

$\nabla^{2} p^{n+1}=\frac{\rho^{n} \nabla \cdot \vec{u}^{* *}}{\Delta t}$

In these two equations, the computed pressure field will be consistent with the requirement for incompressible flow fields that the velocity fields be divergence-free. It is, however, an advantage to have density included at a partially updated time as in Eqn. (32) instead of being evaluated at old time as in the MAC-type Eqn. (33). Moreover, in the nearly incompressible limit Eqn. (28) additionally allows for potentially important spatial gradients in the density field to drive the pressure Poisson equation, and thus to be coupled with the pressure and velocity fields. This could be important, for example, in natural convection flows. It is apparent that if a primitive variable strictly incompressible approach is adopted along with a Boussinesque approximation, the only way bouyant forces could enter the pressure Poisson equation is via the velocity divergence term in Eqn. (32), which in turn comes from an explicit velocity (momentum) predictor equation. Unlike the nearly incompressible limit Poisson Eqn. (28), the density gradient effects would then be neglected.

\subsection{Fully Compressible Limit}

On the other end of the spectrum, for fully compressible flows $\tau_{u}>\tau_{c}>\Delta t$, or $c^{* *} \Delta t<<l$, and the pressure Poisson Eqn. (27) can be rewritten as

$\frac{1}{\left(\frac{\partial f}{\partial \rho}\right)^{* *}} \frac{p^{n+1}-p^{n}}{\Delta t}-\frac{\left(\frac{\partial f}{\partial e}\right)^{* *}}{\left(\frac{\partial f}{\partial \rho}\right)^{* *}} \frac{e^{* *}-e^{n}}{\Delta t}=-\nabla \cdot(\rho \vec{u})^{* *}$

(as seen from Eqn. (17)) which is effectively a density-based compressible flow algorithm since it is easily recognized that the left side of this equation is an approximation for the term $\frac{\partial \rho}{\partial t}$. In fact, the combination of fractional step 1 above with pressure Eqn. (34) and the correction Eqns. (22)-(25), to which the PCICE methods reduces in this limit, constitutes an explicit, predictor-corrector algorithm for fully compressible flows.

\section{Examples}

We give here three example simulations computed with PCICE-FEM in two spatial dimensions, one for each of the three limiting classes discussed above: strictly incompressible, nearly incompressible, and fully compressible. For the strictly incompressible regime we utilize the thermally driven flow in a square cavity. A von Karman vortex street behind a cylinder will be used as an example illustrating the nearly incompressible regime. For the fully compressible regime we simulate an intense spherical explosion, sometimes known as the Sedov blast wave problem. 


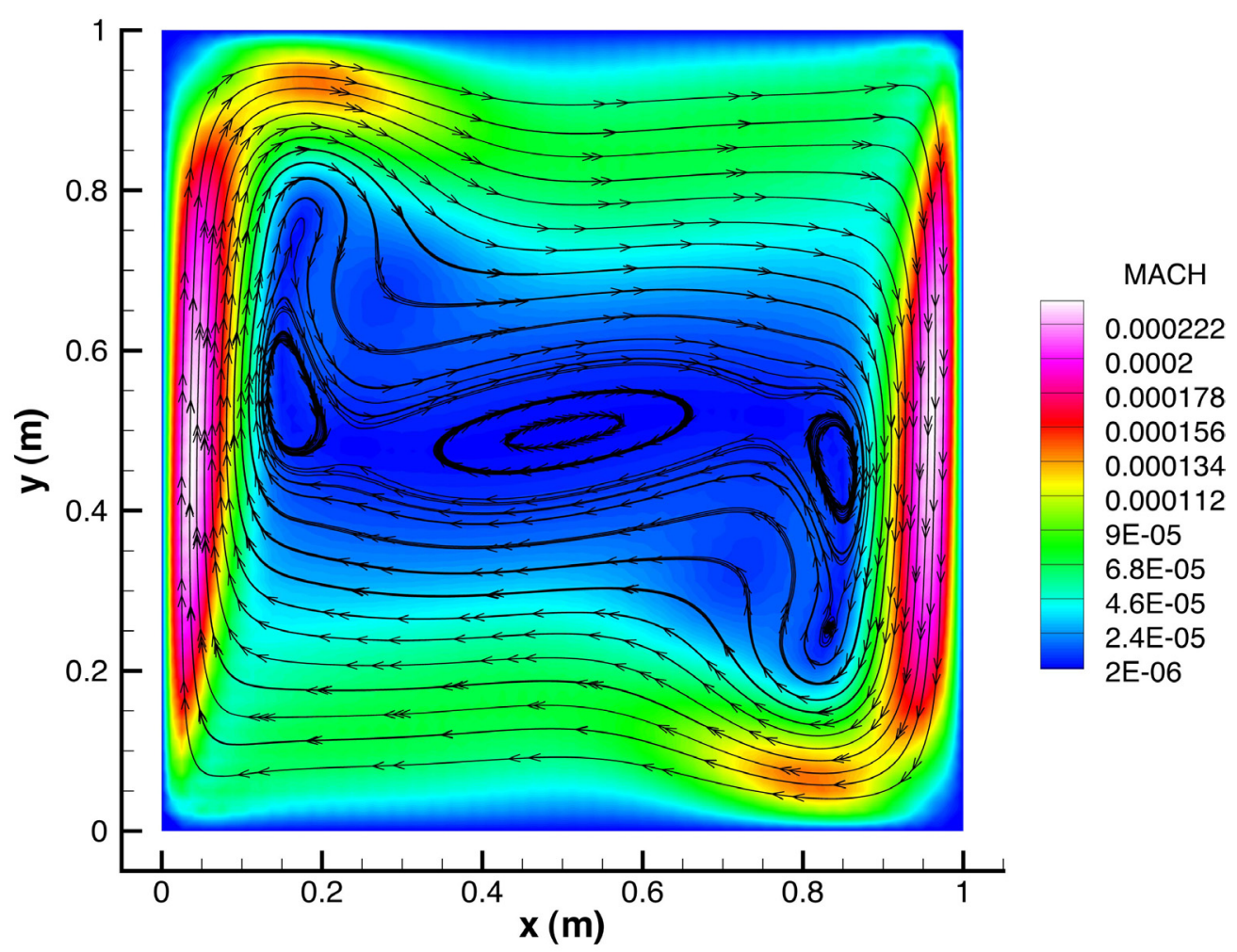

Figure 1. THERMALLY DRIVEN CAVITY, Ra $=10^{6}$, Wall Temperature Difference $=12 \mathrm{~K}$.

\subsection{Strictly Incompressible Example}

We consider the thermally driven, circulatory flow of air in an upright square domain (Heuveline, 2003). The two horizontal walls are defined as no-slip, adiabatic solid walls and the two vertical walls are defined as no-slip isothermal walls, $606 \mathrm{~K}$ on the left wall and $594 \mathrm{~K}$ on the right. Fig. 1 shows the Mach number isolines (color shading) along with the streamline contours for a Raleigh number of $10^{6}$. This agrees very closely with the results obtained by (Heuveline, 2003) as well as those obtained assuming strictly incompressible flow with the Boussinesq approximation. Note, however, that if we changed the temperatures, e.g. to $960 \mathrm{~K}$ and $240 \mathrm{~K}$ keeping the Raleigh number the same (thus, viscosity must be raised), the heat flux would be much higher and density gradients would play a much more dominant role. Then the flow would be within the nearly incompressible regime, and the results would not agree with those obtained assuming incompressible flow with the Boussinesq approximation. This case is also shown in (Heuveline, 2003).

\subsection{Nearly Incompressible Example}

A common benchmark problem for transient algorithms employing the incompressible Navier-Stokes equations is the low Reynolds number flow around a cylinder in a cross flow. Beyond a Reynolds number of 40, the solution becomes unsteady and a periodic shedding of vortices occurs, known as the von Karman vortex street. These vortices are laminar for Reynolds numbers up to approximately 5000. Here we use our compressible, all-speed PCICE-FEM formulation with a free stream velocity equivalent to a Mach number of $\mathrm{M}=0.05$. For a free stream temperature of $300 \mathrm{~K}$ and a free stream pressure of $101325 \mathrm{~Pa}$, the free stream velocity is $17.36 \mathrm{~m} / \mathrm{s}$. The top and bottom walls are set to free-slip. Fig. 2 shows an instantaneous computed Mach number distribution. At this same time, the corresponding instantaneous computed temperature distribution is shown in Fig. 3. Note the small Mach number and the small temperature differences, due to the slight compressibility effects, are resolved on the vortex street, even though the flows are very slow. An incompressible solution would not be able produce this result. 


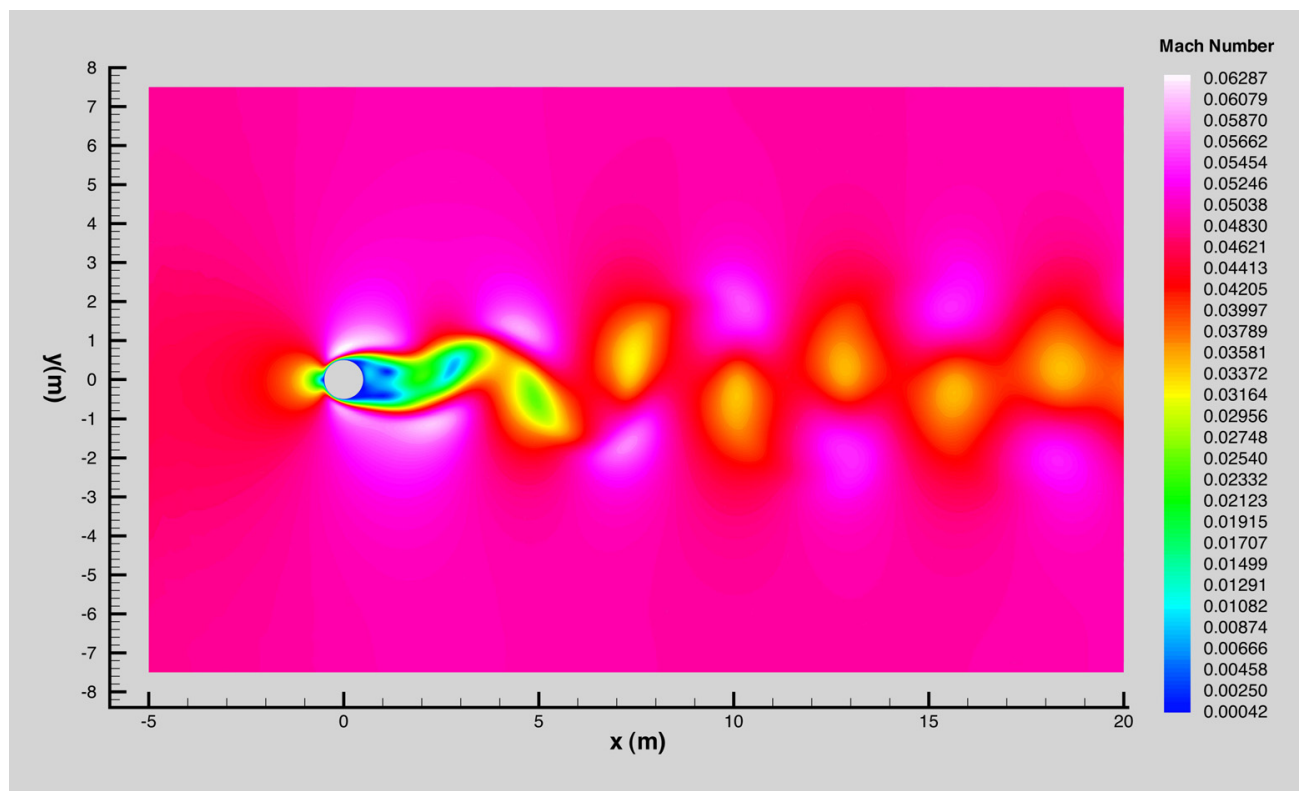

Figure 2. VON KARMAN VORTEX STREET MACH NUMBER SOLUTION, time=4.0 s.

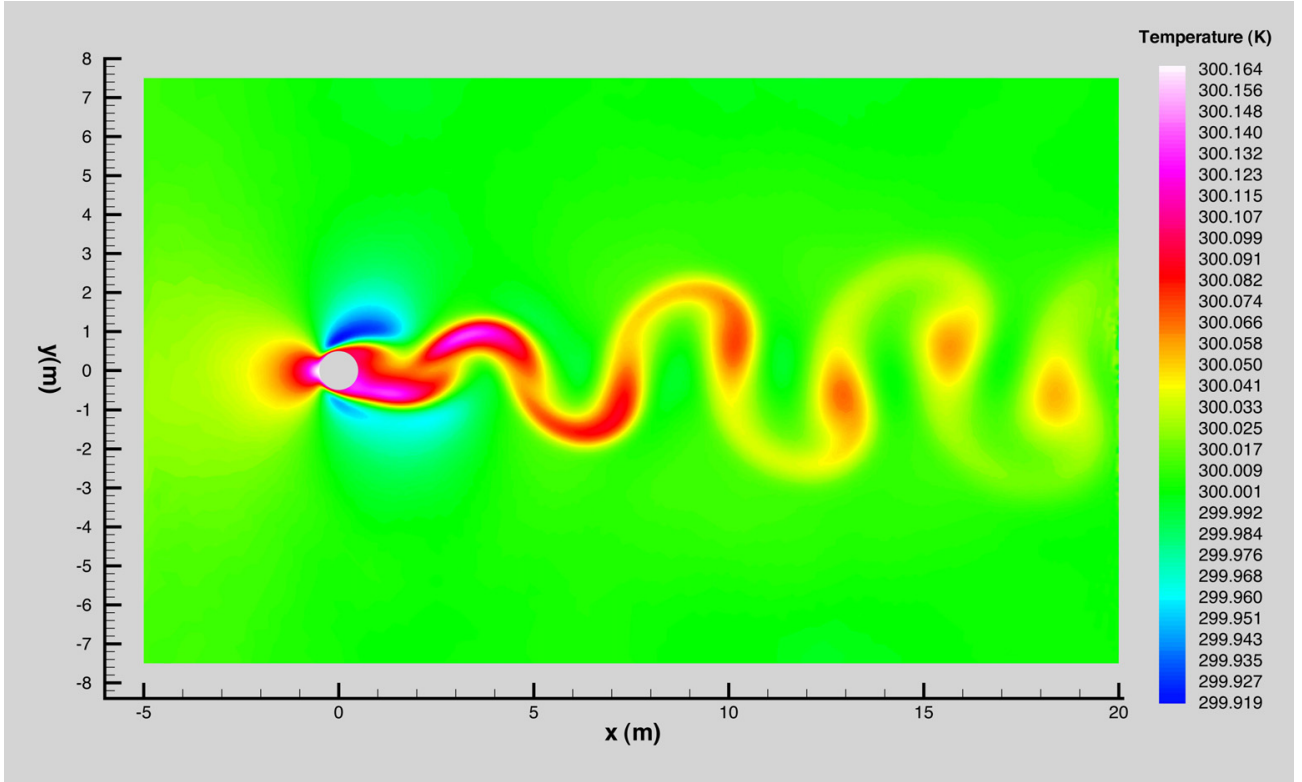

Figure 3. VON KARMAN VORTEX STREET TEMPERATURE SOLUTION, time $=4.0 \mathrm{~s}$.

\subsection{Fully Compressible Example}

The Sedov blast wave (Sedov, 1959) problem is characterized as an intense point explosion in a uniform ideal gas, initially at rest, with negligible initial pressure and finite initial density. This axisymmetrical PCICE-FEM (Martineau, 2007) simulation incorporates the initial conditions of the 1945 Trinity fission bomb in New Mexico and is compared to Sedov's analytical solution. A $100 \mathrm{~m}$ radius hemispherical domain is represented by one quadrant of a circular region integrated about the axis of symmetry (x-axis). The estimated explosion energy of the Trinity test is $7.19 \times 10^{13} \mathrm{~J}$. To approximate this explosion, $3.595 \times 101^{3} \mathrm{~J}$ (one-half the explosion energy for one-half of the sphere) is instantly deposited in the finite elements with an elemental distance less than $4.5 \mathrm{~m}$ from the origin. The balance of the domain is at atmospheric conditions, where pressure is initially set to $P=9.41831 \times 10^{10} \mathrm{~Pa}$ and temperature is initially set to $T=2.62497 \times 10^{8} \mathrm{~K}$. This constitutes a 941,831 to 1 drop in pressure for the first time step. The Sedov analytical solution for this explosion energy instantly deposited at the origin with an initial density of $\rho_{0}=1.25 \mathrm{~kg} / \mathrm{m}^{3}$ results in a shock wave location of $84.57 \mathrm{~m}$ from the origin at $8.0 \times 10^{-3} \mathrm{~s}$ after the explosion 
is initiated. Fig. 4 shows computed density distribution $\left(\rho / \rho_{0}\right)$ at this time and Fig. 5 shows a comparison of this density and the pressure solutions with Sedov's analytical solutions along the coordinate axis. Both compare very favorably with Sedov's solution.

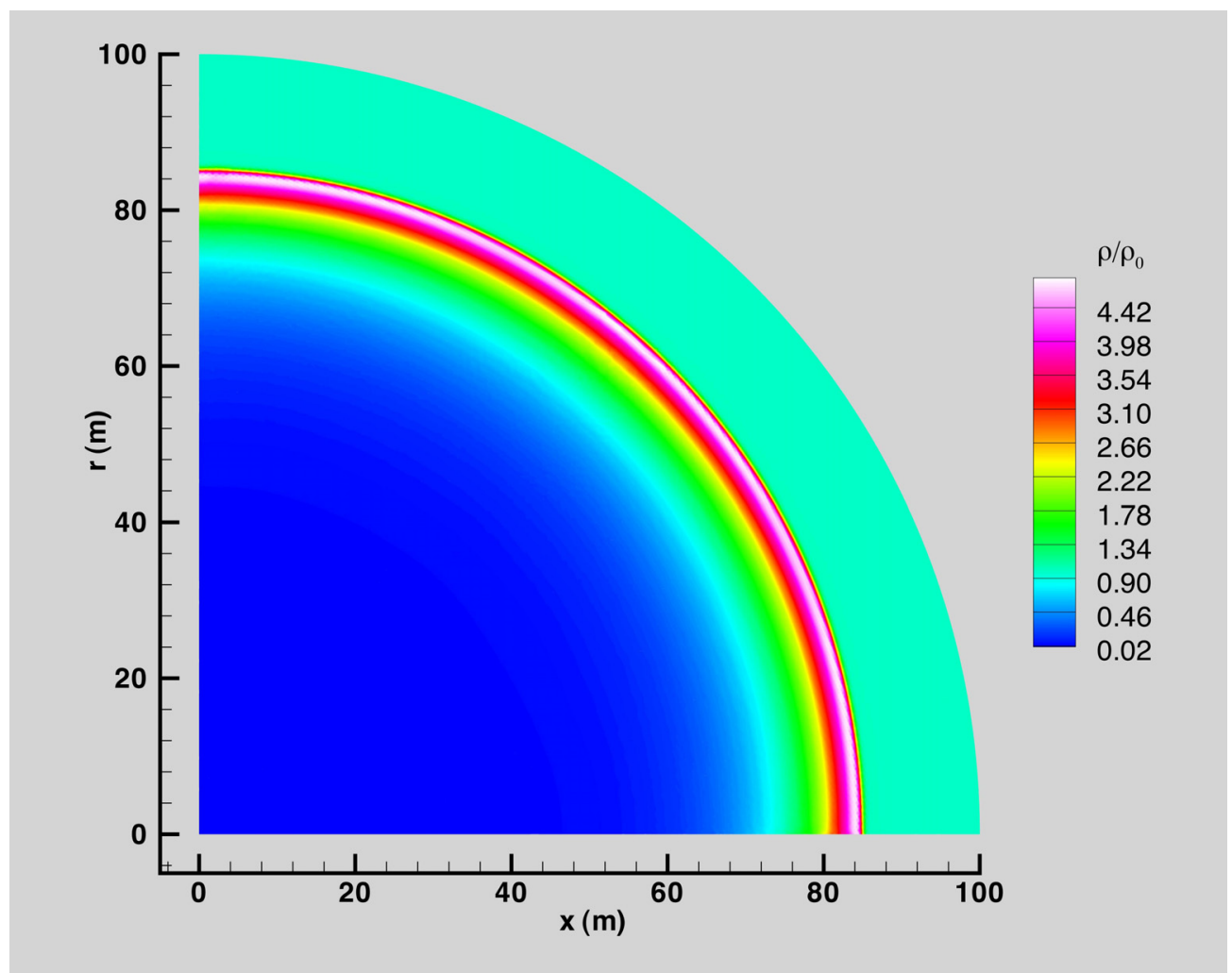

Figure 4. SEDOV BLAST WAVE MASS DENSITY SOLUTION, time $=8.0 \times 10^{-3} \mathrm{~s}$.
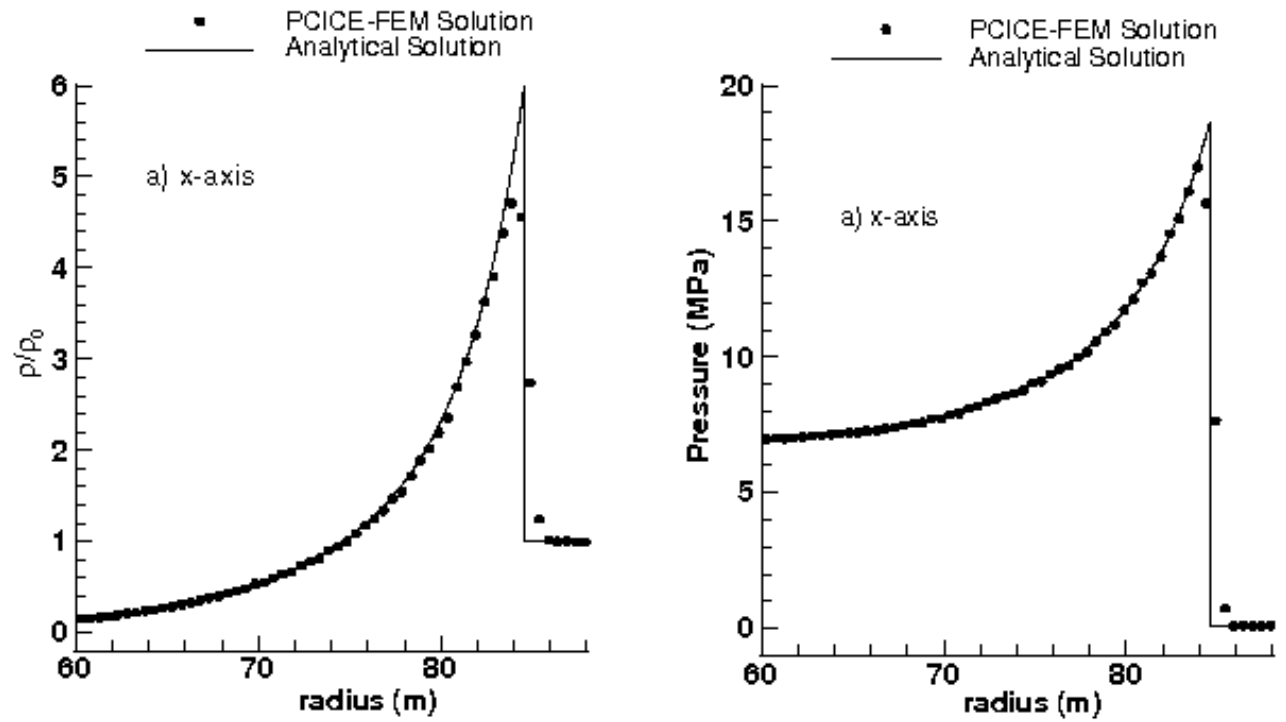

Figure 5. SEDOV BLAST WAVE COMPUTED DENSITY AND PRESSURE COMPARISON TO ANALYTICAL, time $=8.0 \times 10^{-3} \mathrm{~s}$.

\section{Conclusions}

The PCICE method has been shown to reduce to a variant of the traditional MAC-type method for strictly incompressible flows. However, most single phase flows of practical interest in nuclear energy systems do not fall within this narrowly defined restrictive class. Rather, they can be classed as nearly incompressible or fully compressible. For nearly 
incompressible flows (e.g. in natural convection flows where heat flux is significant the strictly incompressible flow assumption coupled with a Boussinesq approximation is physically unrealistic) the PCICE method has been shown to reduce to an appropriate generalization in which density gradients directly serve as a driving mechanism in the flow field. Unlike most other pressure-based methods which claim to allow for compressibility effects, the PCICE method is formulated in conservative form so that supersonic flows and flows containing strong shock waves can be easily considered. The PCICE method has been shown to approximate a conservative-form density-based method for such fully compressible flows. Since the method automatically treats a continuous transition or continuum of compressibility, mixed flows with low speed, even incompressible, in one region and high speed or shock waves in another can be treated.

\section{Acknowledgment}

The author acknowledges the US Department of Energy, Office of Nuclear Energy for funding the preparation of this manuscript at the Idaho National Laboratory under DOE Idaho Operations Office Contract DE-AC07-05ID14517.

\section{References}

Martineau, R.C. and Berry, R.A., The pressure-corrected ICE finite element method (PCICE-FEM) for compressible flows on unstructured meshes, J. Comput. Phys. 198 (2004) 659.

Berry, R.A., Notes on the PCICE method: simplification, generalization, and compressibility properties, J. Comput. Phys. 215 (2006) 6.

Harlow, F.H. and Amsden, A.A., A numerical fluid dynamics calculation for all flow speeds, J. Comput. Phys. 8 (1971) 197.

Lax, P. and Wendroff, B., Systems of conservation laws, Comm. on Pure and Applied Math. 13 (1960) 217.

Leveque, R.J., Numerical Methods for Conservation Laws, Birkhauser Verlag, Basel, 1990.

Casulli, V. and Greenspan, D., Pressure method for the numerical solution of transient, compressible fluid flow, Int. J. for Num. Meth. in Fluids 4 (1984) 1001.

Liles, D.R. and Reed, W.H., Semi-implicit method for two-phase fluid dynamics, J. Comput. Phys. 26 (1978) 390.

Westbrook, C.K., A generalized ICE method for chemically reactive flows in combustion systems, J. Comput. Phys. 28 (1978) 67.

Xiao, F., et al., An algorithm for simulating solid objects suspended in stratified flow, Computer Phys. Comm. 102 (1997) 147.

Patnaik, G., et al., A barely implicit correction for flux-corrected transport, J. Comp. Phys. 71 (1987) 1 .

Swanson, R.C. and Turkel, E., On central-difference and upwind schemes, J. Comput. Phys. 101 (1992) 297.

Harlow, F.H. and Welch, J.E., Numerical calculation of time-dependent viscous incompressible flowof fluids with free surface, Phys. Fluids 8 (1965) 2182.

Heuveline, V., On higher-order mixed FEM for low Mach number flows: application to a natural convection benchmark problem, Int. J. Numer. Meth. Fluids 41 (2003) 1339.

Sedov, L.I., Similarity and Dimensional Methods in Mechanics, Academic Press, New York, 1959.

Martineau, R.C., The PCICE-FEM scheme for highly compressible axisymmetric flows, Int. J. Comput. Fluids, accepted for publication. 